\title{
资源受限的无线传感器网络基于衰减信道的决策融合 ${ }^{*}$
}

李燕君, 王 智 + , 孙优贤

(工业控制技术国家重点实验室(浙江大学),浙江 杭州 310027)

\section{Decision Fusion Under Fading Channel in Resource-Constrained Wireless Sensor Networks}

\author{
LI Yan-Jun, WANG Zhi ${ }^{+}, \quad$ SUN You-Xian
}

(State Key Laboratory of Industrial Control Technology (Zhejiang University), Hangzhou 310027, China)

+ Corresponding author: Phn: +86-571-85698532, E-mail: wangzhi@iipc.zju.edu.cn, http://www.sensornet.cn

Li YJ, Wang Z, Sun YX. Decision fusion under fading channel in resource-constrained wireless sensor networks. Journal of Software, 2007,18(5):1130-1137. http://www.jos.org.cn/1000-9825/18/1130.htm

\begin{abstract}
Decision fusion rules under fading channel in wireless sensor networks are investigated in this paper. Local decisions made by local sensor nodes may be lost or corrupted while transmitted to the fusion center via a fading channel. A series of fusion rules are proposed under the assumption of Rayleigh channel model. Likelihood ratio rule has been shown optimal through theoretical analysis and simulation. However, it consumes system resource and requires good knowledge of local and channel information, which is not easily available in resource-constrained sensor networks. Three sub-optimal alternatives are proposed, which have less computation and information cost. They perform well in their respective SNR (signal-to-noise ratio) range. Finally, it is found that in resource-constrained wireless sensor networks, a tradeoff should be considered among performance, resource cost and computation complexity while choosing the fusion rules.
\end{abstract}

Key words: wireless sensor network; fusion rule; resource-constrained; SNR (signal-to-noise ratio); performance; resource cost; computation complexity

摘 要: 研究了无线传感器网络中衰减信道下的决策融合规则. 由于信道衰减, 由节点传输到融合中心的本地决 策会丢失或产生差错, 要求融合中心的融合规则能够结合信道模型作出最优判决. 在 Rayleigh 分布的信道模型下, 对 一系列融合算法作了理论和仿真分析. 似然比融合算法性能最优, 但是它占用的系统资源大, 需要预知的信息多, 性 价比不高, 不适合资源受限的无线传感器网络.提出了 3 种次优算法,它们比似然比规则耗费的信息代价要小. 在不同 的信噪比(signal-to-noise ratio, 简称 SNR)范围下, 它们的性能有各自的优劣. 综合分析发现, 在资源受限的无线传感器 网络中,最终选择的融合规则应在性能、耗费资源量和复杂度之间获得折衷.

关键词: 无线传感器网络;融合规则;资源受限;信噪比;性能;资源耗费;算法复杂度

中图法分类号: TP393 文献标识码: A

* Supported by the National Natural Science Foundation of China under Grant No.60434030 (国家自然科学基金); the National High-Tech Research and Development Plan of China under Grant No.2006AA01Z218 (国家高技术研究发展计划(863)); the National Research Foundation for the Doctoral Program of Higher Education of China under Grant No.20050335020 (国家教育部博士点基金)

Received 2006-12-31; Accepted 2007-03-05 
无线传感器网络由大量能够感知环境参数(如温度、湿度、压力、可见光与红外线、声音、磁场、加速度 等)的节点组成.这些节点具有一定的计算能力,通过无线方式自组织成网络,具有群体智能,能够协同完成诸如 环境监测、目标跟踪等各类任务,在环境、农业、工业、医疗及商业领域都有广泛应用.

分布在监测区域的传感器节点由于与目标之间的距离各不相同, 单个传感器检测到的相关环境参数并不 能直接反映目标的真实物理状态, 据其作出的判断容易产生虚警, 如果将各个传感器所有的观测信息都直接传 输到用户又会造成网络资源的浪费. 为了提高对目标的识别和估计能力, 降低传输消耗的能量, 可以利用融合技 术, 对分布在不同位置的多个传感器节点提供的局部不完整观测量进行数据融合, 从而清除多传感器之间可能 存在的冗余和矛盾 ${ }^{[1]}$,形成对系统相对一致的感知描述,同时还可以降低网络能耗.

分布式检测融合系统可以采用多种不同的系统配置结构,如并行结构、串行结构、树形结构及网络结构等. 传统的分布式检测算法主要集中在 Bayes 判决及 Neyman-Pearson 判决的研究上. 在传感器观测独立的条件下, 最优判决规则是似然比判决规则; 而在传感器观测相关的条件下, 最优判决规则无法简化为简单的似然比门限 判决.为简化问题的复杂性, 融合算法通常都假设传感器观测独立 ${ }^{[2]}$. 目前, 已有一些文献研究了基于通信限制的 分布式检测算法.Chamberland 等人 ${ }^{[3]}$ 研究发现, 在特定条件下, $N$ 个节点的传感器网络如果通信量限制在单位时 间 $N$ 个比特,那么每个节点发送 1 个比特是最优的.Yuan 等人 ${ }^{[4]}$ 提出了基于窗口的拥塞管理方法来解决本地检 测器与融合中心的通信拥塞问题. Aldosari 等人 ${ }^{[5]}$ 采用非渐近法研究了传感器网络融合问题中多个参数的折衷 问题,包括节点数目、量化度和信噪比(signal-to-noise ratio, 简称 SNR)等.上述文献虽然考虑了通信的限制问题, 但是没有考虑到无线信道的衰减,信息可能发生丢失和差错. 在传感器网络中, 我们选择的融合算法不能过于复 杂,要针对传感网自身特点的应用需求,设计简单且易于操作的融合算法.

本文在 Rayleigh 分布的信道模型下对一系列融合规则作了理论和仿真分析,综合比较了这几种融合规则 的性能、耗费资源量和计算复杂度, 提出了在资源受限的传感器网络中采用的融合规则应当获得上述指标的 折衷.

\section{1 网络场景和系统模型}

在引入抽象的系统模型之前,我们设想了以火灾检测为例的网络场景.在一片区域内部署大量传感器节点, 在火灾发生初始, 周围的节点会检测到某些参数发生变化, 如温度、湿度、光强(烟尘的形成)、红外和紫外辐射 扩散 (火焰)以及气体的释放 ( $\mathrm{CO}, \mathrm{CO}_{2}$ 等). 在实际中, 对某一物理现象的判断常常要同时考虑多个参数的变化, 这 就涉及到多传感器多参数融合的问题. 为了简化研究复杂度, 文中只考虑单个参数的检测问题. 以温度参数的检 测为例,假设已知被监测区域 5 月 9 月的温度如下:

- 绝对温度最高值: $35^{\circ} \mathrm{C}$

- 绝对温度最低值: $8^{\circ} \mathrm{C}$

- 平均温度最高值: $25.5^{\circ} \mathrm{C}$

- 平均温度最低值: $15.5^{\circ} \mathrm{C}$

那么, 单个传感器如果检测到以下情况, 则认为是有火灾发生:

- 某一时刻的温度高于 $45^{\circ} \mathrm{C}$

- 1 分钟内温度变化大于 $10^{\circ} \mathrm{C}$

由于火灾发生的范围内各点的温度和温度变化不同,因此部署在周围的单个节点对火灾是否存在的判断 会有偏差; 可能由于阳光直射使某些点温度骤升, 单个节点会误判为火灾发生而产生虚警. 为避免上述情况的发 生,我们采用多传感器并行结构进行分布式判决.

基于衰减信道的并行结构分布检测系统如图 1 所示, $H_{1}$ 表示有火灾发生, $H_{0}$ 表示无火灾发生. $N$ 个局部节点 在收到未经处理的原始温度数据 $x_{1}, x_{2}, \ldots, x_{N}$ 之后, 在局部节点分别作出局部的二元判决 $u_{1}, u_{2}, \ldots, u_{N}$. 这些局部判 决通过无线信道传输到融合中心. 在向融合中心传输的过程中, 由于信道存在噪声, 信号产生一定的衰减和误 差, 检测判决变为 $y_{1}, y_{2}, \ldots, y_{N}$, 然后, 它们在融合中心得到全局判决 $u_{0}$. 下面我们分 3 个层次来描述这一系统的各 
个参数.

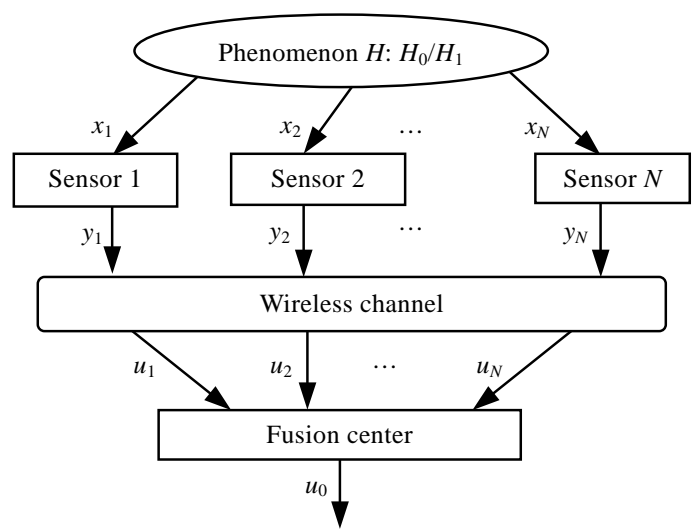

Fig.1 Paralleled distributed detection system under fading channel

图 1 基于衰减信道的并行结构分布式检测系统

局部判决层:第 $k$ 个节点的判决 $u_{k} \in\{+1,-1\}, u_{k}=1$ 表示节点 $k$ 判决 $H_{1}$ 为真, $u_{k}=-1$ 表示节点 $k$ 判决 $H_{0}$ 为真. 检测及虚警概率分别为 $P_{d k}, P_{f k}$, 有 $P_{d k}=P\left[u_{k}=1 \mid H_{1}\right], P_{f k}=P\left[u_{k}=1 \mid H_{0}\right]$.

无线信道层: 无线信道是有信号衰减和噪声的非理想信道.假设信号单调衰减且带有高斯噪声, 这样, $y_{k}=r_{k} u_{k}+n_{k}$. 其中, $r_{k}$ 服从 Rayleigh 分布, 其概率分布函数为 $f\left(r_{k}\right)=\frac{r_{k}}{b^{2}} \mathrm{e}^{-\frac{r_{k}^{2}}{2 b^{2}}}=2 r_{k} \mathrm{e}^{-r_{k}^{2}}, r_{k} \geq 0$, 参数 $b=1 / \sqrt{2}$, 将传输信 号的平均能量标准化, 即 $E\left(r_{k}^{2}\right)=1$, 可得 $E\left(r_{k}\right)=b \sqrt{\pi / 2}=\sqrt{\pi} / 2$. 噪声 $n_{k}$ 服从均值为 0 , 方差为 $\sigma^{2}$ 的高斯分布. 这 样,信噪比就可以简化为 $S N R=10 \log \left(1 / \sigma^{2}\right) \mathrm{dB}$.

融合中心层:基于输入的 $y_{k}$ 和某些系统参数,融合中心根据预设的融合规则得到全局判决 $u_{0}$, 从而判断出监 测区域是否有火灾发生. 融合系统的检测及虚警概率分别为 $P_{D}$ 和 $P_{F}$, 有 $P_{D}=P\left[u_{0}=1 \mid H_{1}\right], P_{F}=P\left[u_{0}=1 \mid H_{0}\right]$. 根据融合 系统的 ROC 曲线, 即检测概率与虚警概率的关系曲线以及系统检测概率随信噪比变化的关系曲线, 我们可以评 价融合规则的检测性能.

\section{2 融合规则}

\section{1 最优融合规则}

假设单个传感器的判决规则已经确定, 则使系统的 Bayes 风险达到最小的最优判决规则是融合中心观测量 的似然比判决,即

$$
\Lambda 1=\log \frac{p\left(y \mid H_{1}\right)}{p\left(y \mid H_{0}\right)}=\log \prod_{k=1}^{N} \frac{P_{d k} \mathrm{e}^{-\frac{\left(y_{k}-r_{k}\right)^{2}}{2 \sigma^{2}}}+\left(1-P_{d k}\right) \mathrm{e}^{-\frac{\left(y_{k}+r_{k}\right)^{2}}{2 \sigma^{2}}}}{P_{f k} \mathrm{e}^{-\frac{\left(y_{k}-r_{k}\right)^{2}}{2 \sigma^{2}}}+\left(1-P_{f k}\right) \mathrm{e}^{-\frac{\left(y_{k}+r_{k}\right)^{2}}{2 \sigma^{2}}}}=\sum_{k=1}^{N} \log \frac{P_{d k} \mathrm{e}^{-\frac{\left(y_{k}-r_{k}\right)^{2}}{2 \sigma^{2}}}+\left(1-P_{d k}\right) \mathrm{e}^{-\frac{\left(y_{k}+r_{k}\right)^{2}}{2 \sigma^{2}}} P_{f k} \mathrm{e}^{-\frac{\left(y_{k}-r_{k}\right)^{2}}{2 \sigma^{2}}}+\left(1-P_{f k}\right) \mathrm{e}^{-\frac{\left(y_{k}+r_{k}\right)^{2}}{2 \sigma^{2}}}}{>}>_{H_{0}}^{>} \eta_{1},
$$

其中, $\eta_{1}$ 为判决门限.

似然比判决 $\Lambda 1$ 需要融合中心预先知道各个节点的性能参数和信道状态信息的瞬时值,虽然理论上它的性 能最优,但在实际操作中占用的系统资源较大,因此,我们需要寻找一些性能次优但占用资源少的替代规则.

\section{2 次优融合规则}

在似然比融合规则的基础上,我们分别对 SNR 较大和 SNR 较小时的情况进行讨论,得出两种简化的融合规 则. 这两种融合规则比最优融合规则需要预知的信息少, 占用资源少, 在 SNR 较大和较小时能使系统性能分别达 到次优.最后,我们还引入一种更为简化的融合规则, 需要的信息量最少,且无论 SNR 大小如何,都能获得较为折 衷的性能.下面, 我们分别用理论加以描述并证明这几种次优融合规则. 
定理 1. 当 $S N R \rightarrow \infty$ 时,似然比融合规则 $\Lambda 1$ 可以近似为 $\Lambda 2^{[6]}$,

$$
\Lambda 2=\sum_{y_{k}>0} \log \frac{P_{d k}}{P_{f k}}+\sum_{y_{k}<0} \log \frac{1-P_{d k}}{1-P_{f k}} \underset{H_{0}}{\stackrel{H_{1}}{>}} \eta_{2} .
$$

证明:定义 $S_{+}$为集合 $\left\{k: y_{k}>0\right\}, S_{-}$为集合 $\left\{k: y_{k}<0\right\}$, 当 $S N R \rightarrow \infty$ 时, $\sigma^{2} \rightarrow 0$,

$$
\Lambda 1 \approx \sum_{S_{+}} \log \frac{P_{d k} \mathrm{e}^{\frac{2 y_{k} r_{k}}{\sigma^{2}}}+\left(1-P_{d k}\right)}{P_{f k} \mathrm{e}^{\frac{2 y_{k} r_{k}}{\sigma^{2}}}+\left(1-P_{f k}\right)}+\sum_{S_{-}} \log \frac{P_{d k}+\left(1-P_{d k}\right) \mathrm{e}^{-\frac{2 y_{k} r_{k}}{\sigma^{2}}}}{P_{f k}+\left(1-P_{f k}\right) \mathrm{e}^{-\frac{2 y_{k} r_{k}}{\sigma^{2}}} .}
$$

对于 $k \in S_{+}, \mathrm{e}^{\frac{2 y_{k} r_{k}}{\sigma^{2}}} \gg>1$, 对于 $k \in S_{-}, \mathrm{e}^{-\frac{2 y_{k_{k} r_{k}}}{\sigma^{2}}} \gg>1$, 且两个分式的分子、分母对 $y_{k}$ 均可导, 分母不为 0 , 因此, 应用洛 比达法则可得

$$
\lim _{\sigma^{2} \rightarrow 0} \Lambda 1=\sum_{y_{k}>0} \log \frac{P_{d k}}{P_{f k}}+\sum_{y_{k}<0} \log \frac{1-P_{d k}}{1-P_{f k}}=\Lambda 2 .
$$

融合规则 12 无须知道信道状况, 只需要知道每个节点的 $P_{d k}$ 和 $P_{f k}$. 但是在 SNR 较小的情况下, 由于算法的 近似处理,使 12 的性能会有所降低.

定理 2. 当 $S N R \rightarrow 0$ 时,似然比融合规则 $\Lambda 1$ 可以近似为 $\overline{\Lambda 3}$,

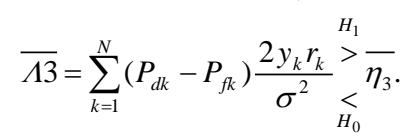

证明:当 $S N R \rightarrow 0$ 时, $\sigma^{2} \rightarrow \infty$,

$$
\Lambda 1 \approx \sum_{k=1}^{N} \log \frac{P_{d k}+\left(1-P_{d k}\right) \mathrm{e}^{-\frac{2 y_{k} r_{k}}{\sigma^{2}}}}{P_{f k}+\left(1-P_{f k}\right) \mathrm{e}^{-\frac{2 y_{k} r_{k}}{\sigma^{2}}}} .
$$

$\mathrm{e}^{-\frac{2 y_{k} r_{k}}{\sigma^{2}}} \rightarrow 1$, 因此, $\mathrm{e}^{-\frac{2 y_{k} r_{k}}{\sigma^{2}}}$ 的一阶泰勒级数近似展开为 $1-\frac{2 y_{k} r_{k}}{\sigma^{2}}$, 有

$$
\lim _{\sigma^{2} \rightarrow 0} \Lambda 1=\sum_{k=1}^{N} \log \frac{P_{d k}+\left(1-P_{d k}\right)\left(1-\frac{2 y_{k} r_{k}}{\sigma^{2}}\right)}{P_{f k}+\left(1-P_{f k}\right)\left(1-\frac{2 y_{k} r_{k}}{\sigma^{2}}\right)}=\sum_{k=1}^{N} \log \left[1-\left(1-P_{d k}\right) \frac{2 y_{k} r_{k}}{\sigma^{2}}\right]-\sum_{k=1}^{N} \log \left[1-\left(1-P_{f k}\right) \frac{2 y_{k} r_{k}}{\sigma^{2}}\right] .
$$

当 $x \rightarrow 0$ 时, $\log (1+x)$ 的一阶泰勒级数近似展开为 $x$, 因此,

$$
\lim _{\sigma^{2} \rightarrow 0} \Lambda 1=\sum_{k=1}^{N}\left[-\left(1-P_{d k}\right) \frac{2 y_{k} r_{k}}{\sigma^{2}}+\left(1-P_{f k}\right) \frac{2 y_{k} r_{k}}{\sigma^{2}}\right]=\sum_{k=1}^{N}\left(P_{d k}-P_{f k}\right) \frac{2 y_{k} r_{k}}{\sigma^{2}}=\overline{\Lambda 3} .
$$

由于 $\sigma^{2}$ 是一个恒定值, 在融合规则中可以略去, 而当节点性质完全相同时, $P_{d k}-P_{f k}$ 也是恒值, 因此, 为了简化 算法,我们不妨假设节点性质是完全相同的,这样, $\overline{\Lambda 3}$ 可进一步简化为 $\Lambda 3$,

$$
\Lambda 3=\frac{1}{N} \sum_{k=1}^{N} y_{k} r_{k} \stackrel{H_{1}}{>} \eta_{H_{0}} \eta_{3} .
$$

其中, 系数 $1 / N$ 是为了下一节应用中心极限定理分析系统性能的需要, 不影响融合规则本身的性质. $\Lambda 3$ 可以直观 地理解为将传输到融合中心的判决加权平均, 权值为信道参数. $r_{k}$ 越大, 信道衰减越小, 相应的判决可信度较高, 给予较大的权值.

在假设每个节点的信道参数也相同时, 我们得到最简化的融合规则 $\Lambda 4$,

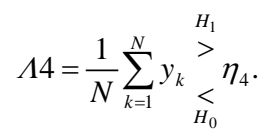


14 可以理解为将所有的判决直接求平均值, 最后与融合中心预先设定的阈值 $\eta_{4}$ 进行比较. 融合中心國值的 选取是另一个值得研究的问题. 文献[7]提出了设计最优阈值的方法, 我们将在以后的文章中予以讨论.

\section{3 仿真及性能分析}

似然比融合规则理论上是性能最优的融合规则 ${ }^{[2]}$, 但是它需要预先知道大量信息,且计算复杂、消耗资源 大. 在资源受限的无线传感器网络中, 我们要尽量简化融合规则, 使做融合的节点消耗的能量尽可能少, 但是也 要满足一定的性能要求. 因此,我们选择的融合规则要在占用资源小、耗能少和性能良好之间获得折衷. $\Lambda 2, \Lambda 3$ 和 $\Lambda 4$ 就是将 $\Lambda 1$ 部分近似得到的 3 种替代融合规则. 在传感器网络中, 为了延长网络寿命, 节点的发射功率通常 保持在中等或较低的水平, 因此, SNR一般不会很高. 由于 $\Lambda 2$ 是 $\Lambda 1$ 当 SNR 较高时得到的近似算法, 所以在传感器 网络中适用的范围不大. 13 和 14 将是值得重点关注的融合算法.

\section{$3.1 \Lambda 3$ 和 $\Lambda 4$ 的 ROC曲线分析}

系统的 ROC 曲线反映系统检测概率与虚警概率的关系.由于上一节我们在引入 13 和 $\Lambda 4$ 时假定各个节点 的性质相同, 可以看作是 $k$ 个独立同分布的判决, 因此, 可以根据中心界限定理对 $\Lambda 3$ 和 $\Lambda 4$ 进行数值逼近. 图 2 是 分别通过 Monte Carlo 仿真和数值逼近得到的 $\Lambda 3$ 和 $\Lambda 4$ 的 ROC 曲线, 其中, $k=10, P_{d}=0.6, P_{f}=0.1$, 信噪比 $S N R=5 \mathrm{~dB}$.

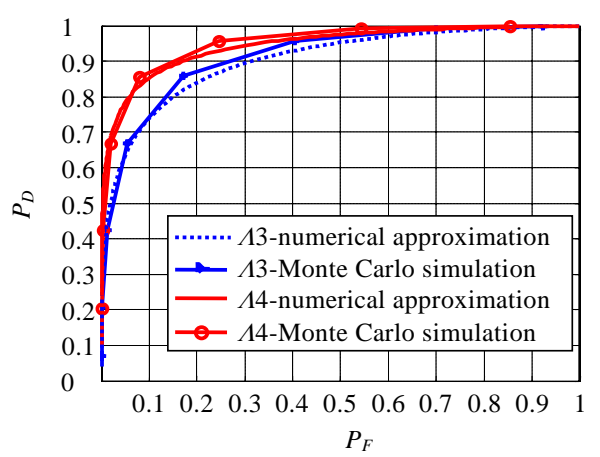

Fig.2 ROC curves for $\Lambda 3$ and $\Lambda 4$ (Monte Carlo simulation and numerical approximation)

图 $2 \Lambda 3$ 和 $\Lambda 4$ 的 ROC 曲线(Monte Carlo 仿真和数值逼近)

从图 2 可以看出, 仿真曲线与数值逼近的曲线有少量偏差, 这是由于所取的 $k$ 不够大, 在满足中心极限定理 的条件上作了一定的放松, 但是曲线整体趋势一致, 吻合程度较为理想. 在相同的虚警概率下, $\Lambda 4$ 的检测概率大 于 $\Lambda 3$, 可见,在 $S N R=5 \mathrm{~dB}$ 时, $\Lambda 4$ 的性能优于 $\Lambda 3$.

\section{2 系统检测概率 $\boldsymbol{P}_{D}$ 与信噪比SNR的关系}

图 3 反映了系统检测概率 $P_{D}$ 与信噪比 SNR 的关系, 其中, 传感器节点数 $k=10, P_{d}=0.6, P_{f}=0.1$, 系统虚警概率 设定为 $P_{F}=0.01$.曲线与第 2 节的理论分析基本一致, 4 种融合算法的性能排序在表 1 中列出. $\Lambda 1$ 性能最优, $\Lambda 2$ 在 SNR 较大时性能较优, $\Lambda 3$ 在 SNR 较小时性能较优, 而 $\Lambda 4$ 在整个 SNR 轴上鲁棒性都不错, 而且 14 占用系统资源 最少, 计算最简便. 在传感器网络中, 我们选择一种融合算法, 其性能固然重要, 但是考虑到传感器网络的特性, 节 省资源包括节省能耗、减少计算量也是我们必须关注的问题.对于不同的应用场合, 系统对融合性能的要求不 同. 如果以性能的保证为首要任务, 那么系统必须牺牲一定的资源满足性能的需要; 如果性能需求一般, 那么要 尽量使系统消耗较少的资源, 以获得更长久的寿命.

为了更直观地比较不同 SNR 取值下 $\Lambda 2, \Lambda 3, \Lambda 4$ 的检测概率与最优融合规则 $\Lambda 1$ 的差别, 我们将每个 SNR 下 它们的差值作了如图 4 所示的曲线. 可以清楚地看到, 在 SNR 较小时, $\Lambda 3$ 与 $\Lambda 1$ 的差别最小, $\Lambda 2$ 与 $\Lambda 1$ 的差别最大; 而在 SNR 较大时, $\Lambda 2$ 与 $\Lambda 1$ 的差别最小, $\Lambda 3$ 与 $\Lambda 1$ 的差别最大; 从整个曲线与横轴围成的面积来看, $\Lambda 4$ 与横轴围 成的面积最小,说明 $\Lambda 4$ 的平均性能较好, 随着 SNR 变化的鲁棒性能最佳. 我们还发现, 3 条曲线都遵循先上升后 
下降的规律, 说明对于每一种次优规则, 它们都有对应的某个 SNR 值,使它们的性能与最优规则偏离最大, 因此, 我们要尽量避免取曲线峰值处的 SNR 值, 在给定 SNR 值的情况下, 所选融合规则的性能要尽量接近最优规则.

Table 1 Performance rating under different signal-to-noise ratio range

表 1 不同信噪比范围下的性能排序

\begin{tabular}{cc}
\hline Range of SNR value $(\mathrm{dB})$ & Performance rating \\
\hline$-20 \sim 10$ & $\Lambda 1>\Lambda 3>\Lambda 4>\Lambda 2$ \\
$-10 \sim 0$ & $\Lambda 1>\Lambda 4>\Lambda 3>\Lambda 2$ \\
$0 \sim 10$ & $\Lambda 1>\Lambda 4>\Lambda 2>\Lambda 3$ \\
$10 \sim 40$ & $\Lambda 1>\Lambda 2>\Lambda 4>\Lambda 3$ \\
\hline
\end{tabular}

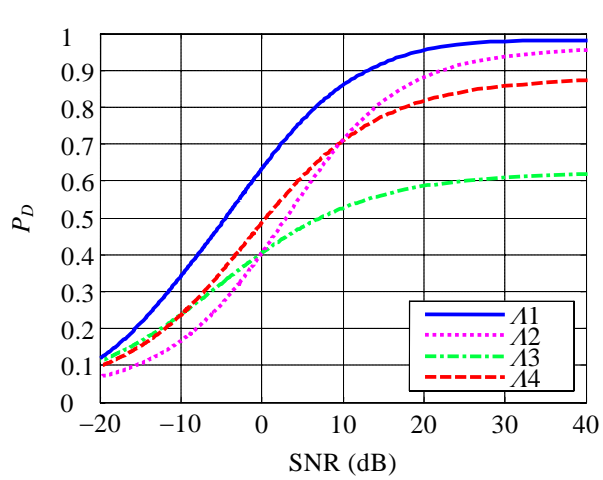

Fig.3 Relationship between system detection probability $P_{D}$ and SNR

图 3 系统检测概率 $P_{D}$ 与 $\mathrm{SNR}$ 的关系

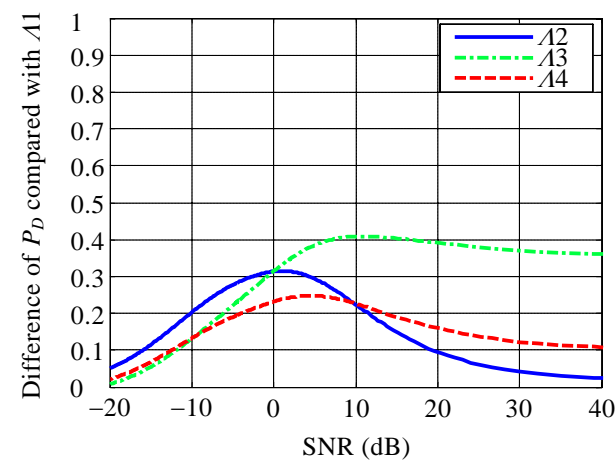

Fig.4 Difference of system detection probability $P_{D}$ ( $\Lambda 2, \Lambda 3$ and $\Lambda 4$ compared with $\Lambda 1)$

图 $4 \Lambda 2, \Lambda 3, \Lambda 4$ 的系统检测概率与 $\Lambda 1$ 的差别

\section{3 偏移系数(deflection coefficient) 分析}

文献[8]中提出了将偏移系数作为评价检测性能的评价标准.它可以被看作系统的输出信噪比, 其值越大, 说 明系统的检测性能越好.其计算方法定义为

$$
D C(\Lambda)=\frac{\left(E_{1}(\Lambda)-E_{0}(\Lambda)\right)^{2}}{V_{0}(\Lambda)} .
$$

其中, $E_{1}$ 和 $E_{0}$ 分别为 $H_{1}$ 和 $H_{0}$ 下融合结果的数学期望, $V_{0}$ 是 $H_{0}$ 下的方差. 使用偏移系数比较系统性能比使用 ROC 曲线要方便, 因为偏移系数计算出来的是一个数值, 而 ROC 为一条曲线. 图 5 为偏移系数与信噪比的关系, 曲线 与图 3 结果类似.

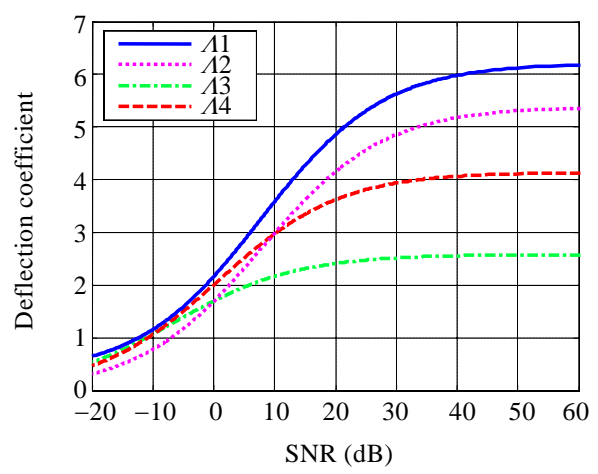

Fig.5 Relationship between deflection coefficient DC and SNR

图 5 偏移系数 DC 与 SNR 的关系 


\section{4 系统检测概率 $\boldsymbol{P}_{D}$ 与节点数 $k$ 的关系}

图 6(a)和图 6(b)反映了当 SNR一定时,系统检测概率与节点数的关系,其中, $P_{d}=0.6, P_{f}=0.1$, 系统虚警概率设 定为 $P_{F}=0.01$. 随着节点数 $k$ 值的增大, 4 种融合算法的系统检测概率都会上升. 当 $k$ 大到一定程度时, 尽管本地传 感器节点的检测性能一般, 但是系统检测概率都能达到 1 , 这是由于大量传感器信息的积累使系统性能得到了 提升.比较图 6(a)和图 6(b) 可以发现, 当 SNR 较大时(如图 6(b)所示), 检测概率随着 $k$ 值上升的速度较快, $\Lambda 2$ 在 SNR 较大时性能较好, 而在 SNR 减小时性能下降,这与图 3 的关系曲线也有一定的对应关系.

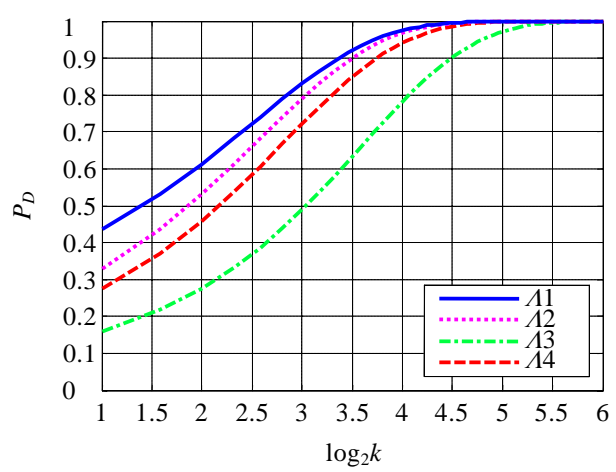

(a) $S N R=2 \mathrm{~dB}$

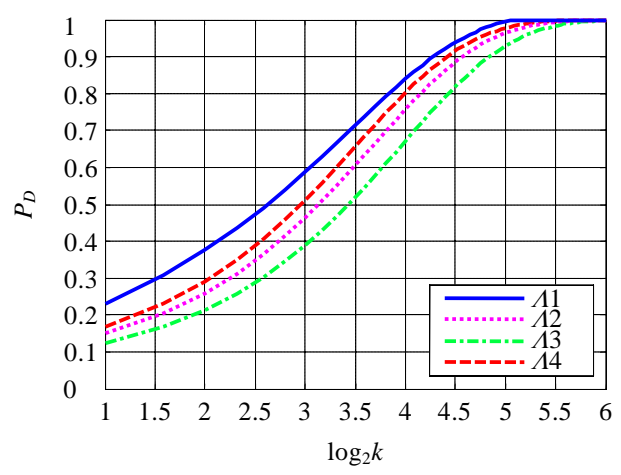

(b) $S N R=12 \mathrm{~dB}$

Fig.6 Relationship between system detection probability and $k$

图 6 系统检测概率与 $k$ 的关系

\section{5 仿真结果小结}

4 种融合算法的假设前提、适用场合以及在性能、占用系统资源和算法复杂度之间的折衷总结于表 2 中. 其中, $\Lambda 2$ 和 $\Lambda 3$ 分别在 SNR 较大和 SNR 较小时性能最接近 $\Lambda 1$, 而 $\Lambda 4$ 在整个 SNR 范围内鲁棒性较好; $\Lambda 1$ 和 $\Lambda 2$ 无任何前提假设,而 $\Lambda 3$ 和 $\Lambda 4$ 有相应的假设; $\Lambda 1$ 需要预知的信息和占用系统资源最大,而 $\Lambda 4$ 几乎不需要预知信 息,占用系统资源最少. 从算法表达式直观上来看, 4 种融合规则的复杂度依次降低, $\Lambda 1$ 最复杂, $\Lambda 4$ 最简单. 由于无 线传感器网络是低功耗的无线网络, 其信噪比水平不可能很高, 且资源受限, 要求算法简单易行, 占用资源少、能 耗低.综上所述, $\Lambda 3$ 和 $\Lambda 4$ 是适合无线传感器网络的融合规则, $\Lambda 3$ 在环境噪声很大时适用, 而 $\Lambda 4$ 由于其较好的鲁 棒性适用于环境噪声变化频繁的网络.

Table 2 Comparison of several metrics of the four fusion rules

表 24 种融合规则的各项指标比较

\begin{tabular}{cccccc}
\hline $\begin{array}{c}\text { Fusion } \\
\text { rules }\end{array}$ & Assumptions & Performance & $\begin{array}{c}\text { Priori information required } \\
\text { and resource consumption }\end{array}$ & $\begin{array}{c}\text { Algorithm } \\
\text { complexity }\end{array}$ & Applied situation \\
\hline$\Lambda 1$ & None & Optimal & $\begin{array}{c}\text { Channel SNR and } \\
\text { node parameters }\end{array}$ & Most complex & Traditional networked sensor \\
\hline$\Lambda 2$ & None & $\begin{array}{c}\text { Suboptimal for } \\
\text { large SNR }\end{array}$ & Node parameters & Complex & $\begin{array}{c}\text { Sensor networks with } \\
\text { small channel noise }\end{array}$ \\
\hline$\Lambda 3$ & Identical nodes & $\begin{array}{c}\text { Suboptimal for } \\
\text { small SNR }\end{array}$ & Channel SNR & Simple & $\begin{array}{c}\text { Sensor networks with } \\
\text { large channel noise }\end{array}$ \\
\hline$\Lambda 4$ & $\begin{array}{c}\text { Identical nodes with the } \\
\text { same channel gain }\end{array}$ & Most robust & None & Most simple & $\begin{array}{c}\text { Sensor networks with frequently } \\
\text { varying channel noise }\end{array}$ \\
\hline
\end{tabular}

\section{4 结束语}

本文研究了无线传感器网络中信道衰减情况下并行分布式检测的决策融合问题,以似然比融合规则为基 础提出了 4 种融合规则. 通过理论分析和仿真实验比较了这几种融合规则的性能,提出了适用于传感器网络的 融合规则不能只追求性能的优越,而要在性能、算法复杂度、占用系统资源大小之间找到折衷. 
考虑无线传感器网络自身特点的融合问题是一个值得研究的领域. 目前, 我们的研究还仅局限于二元判决 的单跳并行网络.混合结构的多元多跳网络的融合规则、融合路径与路由的关系、融合耗费能量的具体量化以 及判决的门限问题都将是我们今后的研究方向.

致谢 在此, 我们向对本文的工作给予支持和建议的同行, 尤其是杭州电子科技大学的柴利老师和浙江大学网 络传感与控制研究组的曹峰同学表示感谢.

\section{References:}

[1] Zhu YM. Multisensor Distributed Statistic Decision. Beijing: Science Press, 2000. 19-21 (in Chinese).

[2] Han CZ. Multi-Source Information fusion. Beijing: Tsinghua University Press, 2006. 195-200 (in Chinese).

[3] Chamberland J, Veeravaili V. Decentralized detection in sensor networks. IEEE Trans. on Signal Processing, 2003,51(2):407-416.

[4] Yuan YQ, Moshe K. Distributed decision fusion with a random-access channel for sensor network application. IEEE Trans. on Instrumentation and Measurement, 2004,53(4):1339-1344.

[5] Aldosari S, Moura J. Fusion in sensor network with communication constraints. In: Proc. of the IEEE/ACM Symp. on Information Processing in Sensor Networks (IPSN 2004). New York: ACM Press, 2004. 108-115. http://ieeexplore.ieee.org/xpls/abs_all.jsp? isnumber $=29021 \&$ arnumber $=1307329 \&$ count $=52 \&$ index $=13$

[6] Chair Z, Varshney PK. Optimal data fusion in multiple sensor detection system. IEEE Trans. on Aerospace Electron System, 1986, 22(1):98-101.

[7] Israfil B, Ghassan A, Yucel A. Parallel distributed detection for wireless sensor networks: Performance analysis and design. In: Proc. of the IEEE Global Telecommunications Conf. (GLOBECOM 2005). Piscataway: IEEE, 2005. 2420-2424. http://ieeexplore.ieee. org/xpls/abs_all.jsp?isnumber=33288\&arnumber=1578097\&count=118\&index=113

[8] Picinbono B. On deflection as a performance criterion in detection. IEEE Trans. on Aerospace Electron System, 1995,31(3): $1072-1081$.

\section{附中文参考文献:}

[1] 朱允民.多传感器分布式统计判决.北京:科学出版社,2000.19-21.

[2] 韩崇昭.多源信息融合.北京:清华大学出版社,2006.195-200.

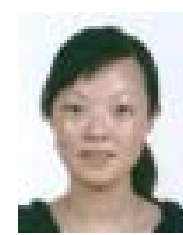

李燕君 $(1982-)$,女,江苏南通人,博士生, 主要研究领域为无线传感器网络的链路 估计,路由算法,数据融合.

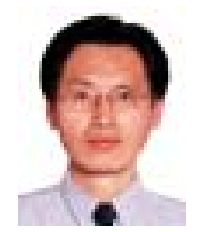

王智(1969-), 男,博士, 副研究员, 博士生 导师, CCF 高级会员,主要研究领域为无线 传感器网络, 实时与工业通信, 网络控制.

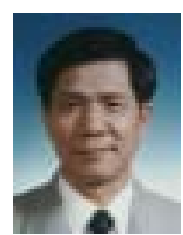

孙优贤(1940 - ), 男, 教授, 博士生导师, 中 国工程院院士, 主要研究领域为复杂工业 过程建模,控制与优化,工厂综合自动化系 统, 大型装备自动化成套系统, 鲁棒控制理 论及应用. 